\title{
Infections: The Emergency of the New Millennium
}

\author{
Nicola Petrosillo
}

\begin{abstract}
"For all they knew, in that invisible micro-organic world there might be as many different kinds of germs as there are grains of sand on this beach. And also, in that same invisible world it might well be that new kinds of germs came to be. It might be there that life originated..."
\end{abstract}

(Jack London. The Scarlet Plague. Mills \& Boon, Limited, London 1916)

\subsection{Introduction}

At the beginning of 2015, the British Economist Jim O'Neill chaired a review of antimicrobial resistance, commissioned by the UK Government and the Wellcome Trust; on May 2016, he published a report titled "Tackling drug-resistant infections globally: final report and recommendations." In this report, the estimate of the burden of antimicrobial resistance in the next decades was shocking. He estimated that "by 2050, 10 million lives a year and a cumulative 100 trillion USD of economic output are at risk due to the rise of drug-resistant infections if we do not find proactive solutions now to slow down the rise of drug resistance. Even today, 700,000 people die of resistant infections every year" [1].

In the same period, a devastating Ebola virus epidemic plagued part of West Africa and threatened developed countries. The Ebola epidemic in the period 20132016 hit 28,616 suspected cases and caused 11,310 deaths [2].

The first two decades of the New Millennium are facing several problems in healthcare. Antimicrobial resistance is spreading worldwide due to antibiotic

\footnotetext{
N. Petrosillo $(\bowtie)$

National Institute for Infectious Diseases "Lazzaro Spallanzani”, IRCCS, Rome, Italy

e-mail: nicola.petrosillo@inmi.it
} 
overuse/misuse in the human and animal setting, and even environment is polluted with resistant organisms and antimicrobials.

While the demand for health is growing all over the world, technology is advancing, surgical interventions are becoming more complex, the availability of drugs and diagnostic and therapeutic aids is increasingly extensive and modern, and the risk of infection acquired in health facilities is becoming too high, with its weight of morbidity, mortality, and costs for the community.

If the risk factors and all the other socio-economical reasons for developing and spreading emerging infections will remain in the next years-and there is no reason why they will not-these infection will represent a challenge for the healthcare system in the New Millennium because hospital and community preparedness is widely insufficient, awareness among professionals and media raises only when an epidemic is ongoing, and prevention is lacking.

\subsection{From Optimism to the Harsh Reality: The History of Infectious Diseases in the Past Century}

The first half of the twentieth century was dominated by an optimistic feeling on the treatment, control, and prevention of infectious diseases.

In 1931, Henry Sigerist wrote, "Most of the infectious diseases ... have now yielded up their secrets.... Many illnesses ... had been completely exterminated; others had ... largely under control...." [3]. Up to 1960, antibiotics were extensively developed by pharmaceutical industries and immunization added to this optimism. All this contributed to the sentence, today ironically mentioned almost in every conference dealing with antimicrobial resistance and emerging infection, by the Surgeon General William H. Stewart to the US Congress that it was time to "close the book on infectious diseases" [4].

All this determined a drastical reduction of funding for the research against infectious diseases. Pharmaceutical companies shut down the development of new antibiotics and only few drops come out of the pipeline. In much of the developed world, the public had forgotten the impact of infectious diseases on previous generations and shared in the confidence that modern medicine and technology would prevail.

In the late 1970s-early 1980s, this atmosphere of optimism was clouded by some dramatic epidemics that changed the feeling and opinion on the future of infectious diseases. Since 1976, an outbreak of Ebola virus disease was spreading in Zaire with a mortality rate up to $88 \%$; however, the emergence of a new disease in United States, namely the Legionnaire disease outbreak at Philadelphia in 1976, was the first sign of something changing between humans and organisms, and of the susceptibility of people with comorbidities to emerging infectious agents. Finally, what completely changed the history of infectious diseases was the emergence of the first cases of pneumocistosis at S. Francisco in 1981, and the subsequent discovery of the human immunodeficiency virus (HIV) that caused opportunistic infections (AIDS) in infected individuals. Other emerging infections occurred worldwide later on, including Hantavirus epidemic in United States in 1993, H5N1 influenza in China in 1993, Diphtheria in Eastern Europe in 1997 after the fall of the barriers from West and East Europe, SARS in Asia in 2003, etc. [5]. 


\subsection{What Are Emerging Infectious Diseases (EIDs)?}

EIDs are not only those infections that create social alarm when the media discover them, they include [6]

- events caused by newly evolved organisms, such as multidrug-resistant tuberculosis, plasmid-mediated colistin resistance in Gram negatives, carbapenemresistant Enterobacteriaceae, etc.

- infections caused by germs entering human population for the first time recently, such as HIV-1 infection in 1981, severe acute respiratory syndrome (SARS) coronavirus in 2003, Middle East respiratory syndrome caused by a novel coronavirus (Middle East respiratory syndrome coronavirus, or MERS-CoV) that was first identified in 2012 in Saudi Arabia

- infections caused by pathogens that are historically present, but with a recent dramatic increase, such as Ebola virus disease in Democratic Republic of Congo in 2018

The main concern for EIDs is represented by their capability of spreading causing epidemic/pandemic and social alarm. Main causes of EID spread include (Fig. 1.1)

- Poverty, famine, displacement, and war all represent conditions that are ruthlessly exploited by infectious diseases and emerging pathogens. Moreover, war events often stop or deteriorate ongoing infection prevention and control pro-

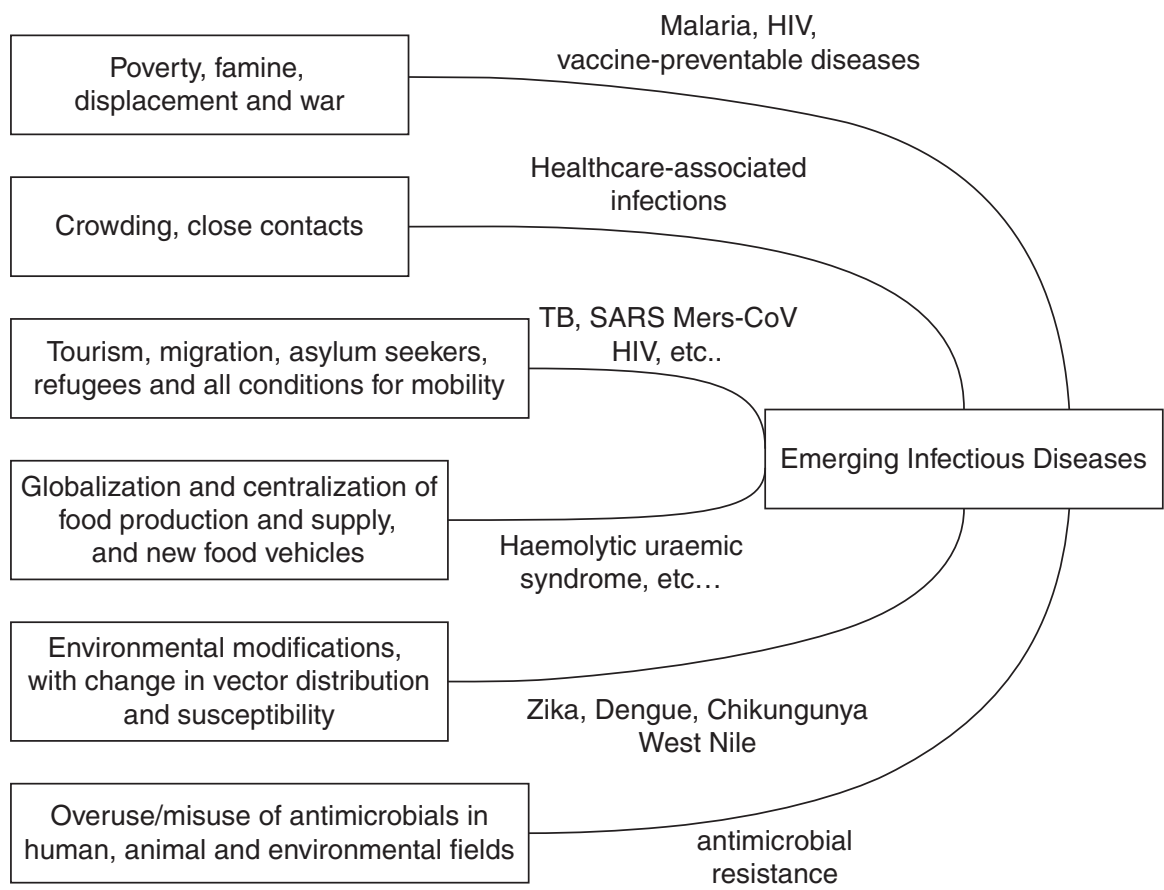

Fig. 1.1 Major drivers for emergence of threatening infections in the New Millennium 
grams, both in the community and healthcare setting, including vaccination, medication supply, access to surgical and medical care, etc. [7]. Re-emergence of malaria by Plasmodium falciparum in Afghanistan, trypanosomiasis in Democratic Republic of Congo, AIDS, vaccine-preventable diseases such as measles, all are dramatically affected by war events [7].

- Crowding: micro-organisms often require close contact for their direct or indirect transmission; this is valid both for community (SARS, MERS-CoV) and healthcare-associated infection. Crowding is a condition that can facilitate the transmission of potentially life-threatening infections because of the likelihood of reaching susceptible hosts and, as it is the case of patients in crowded healthcare facilities, for the easier probability to have limited/poor infection control measures and understaffing.

- Tourism, migration, asylum seekers, refugees, and all conditions for mobility. Travel can model infectious disease dynamics by introducing potential pathogens into susceptible populations or by modifying the rate of contacts and exposures between infected and susceptible individuals.

- Globalization and centralization of food production and supply, and new food vehicles: the case of hemolytic uremic syndrome (HUS) clusters of cases caused by Shiga toxin-producing Escherichia coli (STEC) O157 in Germany in 20162017 likely associated with packaged minced meat consumption is emblematic.

- Environmental modifications, with change in vector distribution and susceptibility. The recent emergence of Zika, the spread of Dengue, Chikungunya, and West Nile fever in countries that was not present in the past clearly document an ecological change involving especially vectors.

- Overuse/misuse of antimicrobials in human, animal, and environmental fields [8].

\subsection{The New Millennium: Infectious Disease Threats}

At the beginning of the New Millennium, the severe acute respiratory syndrome (SARS) coronavirus $(\mathrm{CoV})$ outbreak created social and political alarm because of the threat of transmission of the highly life-threatening virus through international traveling from Asia. The big economical expansion of Asian countries and their international trade seemed to be hampered by the fear of contagion. Luckily, the outbreak was limited and the alert quickly returned. However, this outbreak raised attention to the fact that emerging infections originate largely from animals (zoonosis).

Indeed, SARS was introduced into humans from civet cats sold for consumption at markets, which had acquired infection from the original reservoir, horseshoe bats [9]. This was the first signal that new infections were under the corner. As a matter of concern, the source of EIDs is often from animals (zoonosis), that more commonly have a wildlife, as is the case of Nipah virus in Perak, Malaysia, and SARS in Guangdong Province, China. Indeed, zoonotic viral EIDs represent a major and increasing threat to global health. 
If viral emerging infections represent a concern mainly in the underdeveloped area of the world, the emergence of antimicrobial resistance is a growing problem worldwide but especially in developing and high income countries, where misuse and overuse of antibiotics among humans and in the veterinary setting, including animal feeding, artificially created the selection of resistant strains.

Resistance to antimicrobials is a natural process that occurred before the discovery of antibiotics. In 1931, a sulfa drug, prontosil rubrum, was the first clinically useful antibacterial agent to be discovered. However, prontosil was not the first antibacterial agent to be invented, and humans were not the initial inventors. According to sophisticated genetic analysis, it is clear that bacteria invented antibiotics and consequently induced antibiotic resistance mechanisms somewhere between 2 and 2.5 billion years ago. Bacteria have been killing each other with these weapons, and using resistance mechanisms to protect themselves against these weapons, for 20 million times longer than we have even known that antibiotics exist [10]. This was surprisingly discovered in a deep cave explored in New Mexico in 2011, isolated from the surface of the planet for 4 million years, in which scientists cultured many different types of bacteria from the walls of the caves. Resistance to at least one modern antibiotic was found in many isolated organisms, and some of them were multidrug resistant, even to modern antibiotics [11].

However, nowadays the process for creating resistant organisms has been accelerated because overuse of antimicrobials has increased the rate at which resistance is developing and spreading. Indeed, currently the major drivers behind the occurrence and spread of antimicrobial resistance are represented by the use of antimicrobials in human and veterinary field, and by the transmission of resistant organisms between humans, animals, and environment. On the other side, we lack new drugs to challenge these new superbugs. This results in facing a growing enemy with a largely depleted armory. While antimicrobial overuse/misuse determines ecological pressure on bacteria and contributes to the emergence and selection of AMR, poor infection prevention, and control practices is the main cause for the further spread of these bacteria.

From a microbiological point of view, over the past decades, bacteria with specific clones expressing resistant to several antimicrobials spread worldwide. This is the case of Enterobacteriaceae, mainly Klebsiella pneumoniae resistant to carbapenems with different resistance enzymes including KPC (Klebsiella pneumoniae carbapenemase), NDM-1 (New Delhi Metalloprotease), and other metallobetalactamases. More recent pan-resistant strains, expressing also resistance to polymixins, have been identified as cause of severe diseases; some colistin-resistant strains had a chromosomal resistance, likely induced by antibiotic pressure, but in other cases colistin resistance was linked to mobile elements, plasmids, like MCR-1 and others. This emerging resistance represents an important concern due to its potential for spreading among bacteria of different species; as evidenced recently in China, environment is one of the main sources of these mobile elements, likely due to the wide use of colistin in agriculture [12].

Antimicrobial resistance is commonly found in organisms that cause healthcareassociated infections, but it is not only a matter of hospitals and other healthcare facilities because it is widespread also in the community. 


\subsection{The Healthcare-Associated Infections and Their Burden of Antimicrobial Resistance in the New Millennium}

Healthcare-associated infections (HAI) can be acquired during home, outpatient, long term or hospital care; they represent one of the greatest challenges of modern medicine. In United States, according to the Institute of Medicine, nosocomial infections now concern 5-15 per 100 hospitalized patients and can lead to complications in $25-50 \%$ of those admitted to intensive care units [13]. From 2016 to 2017, a large point prevalence survey of HAI and antimicrobial use was conducted in the European Union and European Economic Area (EU/EEA) including 310,755 patients from 1209 acute care hospitals in 28 countries and 117,138 residents from 2221 long-term care facilities in 23 countries. The authors estimated that $6.5 \%$ (cumulative $95 \%$ confidence interval (cCI): 5.4-7.8\%) patients in acute care hospitals and 3.9\% (95\% cCI: $2.4-6.0 \%$ ) residents in longterm care facilities had at least one HAI. It means that in Europe, on any given day, more than 98,000 patients in acute care hospitals and around 130,000 residents in long-term care facilities had an HAI. The burden of HAI is therefore alarming, with around 9 million infections yearly in acute care hospitals and 4.4 million in long-term care facilities. More than a quarter of HAIs were respiratory tract infections, mainly pneumonia, followed by urinary tract infections (18.9\%), surgical site infections (18.4\%), bloodstream infection (8.9\%), and gastrointestinal infections (8.9\%). Among the latter around half of them were Clostridioides difficile infections.

In this survey, in acute care hospitals the 10 most commonly isolated organisms included E. coli (16.1\%), S. aureus (11.6\%), Klebsiella spp. (10.4\%), Enterococcus spp. (9.7\%), P. aeruginosa (8.0\%), C. difficile (7.3\%), Coagulase negative Staphylococci (7.1\%), Candida spp. (5.2\%), Enterobacter spp. (4.4\%), and Proteus spp. (3.8\%).

Moreover, the prevalence survey assessed the rate of antimicrobial resistance in these infections that is $31.3 \%$ in acute care hospitals and $28 \%$ in long-term care facilities [14].

Rates of antimicrobial resistance in Europe are annually assessed by the European Centers for Disease Control (ECDC). In their last report [15], ECDC well describes the situation of antimicrobial resistance in Europe. For E. coli, more than half $(58.2 \%)$ of the isolates reported to EARS-Net for 2017 were resistant to at least one of the antimicrobial groups under regular surveillance, i.e., aminopenicillins, fluoroquinolones, third-generation cephalosporins, aminoglycosides, and carbapenems. Resistance was the highest for aminopenicillins (58.7\%) and lowest, fortunately, for carbapenems (less than 1\%).

K. pneumoniae is one of the biggest issues in Europe and worldwide. At the EU/ EEA level, more than a third (34.1\%) of the isolates reported to EARS-Net for 2017 were resistant to at least one of the antimicrobial groups under regular surveillance, i.e., fluoroquinolones, third-generation cephalosporins, aminoglycosides, and 
carbapenems. Carbapenem resistance was on average $7.2 \%$, but the range was extremely variable with highest rates, up to $65 \%$, in Southern/Eastern Europe.

Among Gram negatives, Pseudomonas aeruginosa remains one of the most threatening organisms. Less than one third of the isolates in Europe, according to EARS-Net were resistant to at least one of the antimicrobials historically used against these organisms, namely piperacillin/tazobactam, fluoroquinolones, ceftazidime, aminoglycosides, and carbapenems.

The list of challenging Gram negatives is completed by Acinetobacter baumannii, non-fermenting organisms that in more than half of the isolates reported to ERAS-NET were resistant to at least one of the antimicrobials historically used against, namely fluoroquinolones, aminoglycosides, and carbapenems. In some European countries, resistance to carbapenems is up to $96 \%$.

However, the danger does not only come from Gram negatives. Staphylococcus aureus, the most known Gram-positive organism, responsible for sepsis, osteomyelitis, skin and soft tissue infections, surgical site infections, and pneumonia, carries a resistance to methicillin up to $44.4 \%$ in Europe.

\subsection{Conclusions}

Mobility, people traveling much more frequently and far greater distances than in the past, living in more densely populated areas, centralization of food production and supply and new food vehicles, war, famine and displacement, change in vector distribution and susceptibility, and more opportunities to come into closer contact with wild animals, all represent a potential for emerging infectious diseases, either caused by new or well-known viral agents or by antimicrobial-resistant bacteria to spread rapidly and cause outbreaks locally or globally.

Whereas viral emerging infections are marginally a concern for developed countries, that are just lapped by them, antimicrobial resistance is importantly a matter of developed and developing countries, where this issue has become a top priority for global policy makers and public health authorities. New mechanisms of resistance continue to emerge and spread globally, threatening our ability to treat common infections. The overuse and misuse of antimicrobials both in the human and animal field, and their dispersion in the environment pose a risk for selection of mechanisms of resistance of germs. Some settings, like the healthcare facilities, where the use of antimicrobials is obviously intense and infection prevention and control can be poor, represent the reservoir for multidrug-resistant organisms and, sometimes, the melting pot for pan-resistant strains.

The New Millennium opened with alarming data on infections and antimicrobial resistance. Healthcare workers, care givers, decision-makers, politicians, and media should raise public awareness that antimicrobials should be wisely prescribed and administered both in the human and animal field, avoiding self-prescription and overuse of these valuable tools against bacterial infections. 


\section{References}

1. O'Neill J. Tackling drug-resistant infections globally: final report and recommendations; 2016.

2. World Health Organization. http://apps.who.int/gho/data/view.ebola-sitrep.ebola-summarylatest?lang=en. Accessed 9 June 2016.

3. Sigerist HE. The Great Doctors, vol. 372. New York: Dover Publications; 1971.

4. Garrett L. In: Mann JM, Tarantola DJM, Netter TW, editors. AIDS in the World. Cambridge: Harvard University Press; 1992. p. 825-39.

5. Cohen ML. Changing patterns of infectious disease. Nature. 2000;406(6797):762-7.

6. Jones KE, Patel NG, Levy MA, Storeygard A, Balk D, Gittleman JL, Daszak P. Global trends in emerging infectious diseases. Nature. 2008;451(7181):990-3.

7. Conolly MA, Heymann DL. Deadly comrades: war and infectious diseases. Lancet. 2002;360(Suppl):S23-4.

8. Petersen E, Petrosillo N, Koopmans M, ESCMID Emerging Infections Task Force Expert Panel. Emerging infections-an increasingly important topic: review by the Emerging Infections Task Force. Clin Microbiol Infect. 2018;24(4):369-75.

9. Smith KM, Anthony SJ, Switzer WM, Epstein JH, Seimon T, Jia H, et al. Zoonotic viruses associated with illegally imported wildlife products. PLoS One. 2012;7:e29505.

10. Spellberg B, Bartlett JG, Gilbert DN. The future of antibiotics and resistance. N Engl J Med. 2013;368(4):299-302.

11. Bhullar K, Waglechner N, Pawlowski A, Koteva K, Banks ED, Johnston MD, Barton HA, Wright GD. Antibiotic resistance is prevalent in an isolated cave microbiome. PLoS One. 2012;7(4):e34953.

12. Zhou HW, Zhang T, Ma JH, Fang Y, Wang HY, Huang ZX, et al. Occurrence of plasmid and chromosome-encoded mcr-1 in water-borne Enterobacteriaceae in China. Antimicrob Agents Chemother. 2017;61(8)

13. Pittet D. Infection control and quality health care in the new millenium. Am J Infect Control. 2005;33:258-67.

14. Suetens C, Latour K, Kärki T, et al. Prevalence of healthcare-associated infections, estimated incidence and composite antimicrobial resistance index in acute care hospitals and longterm care facilities: results from two European point prevalence surveys, 2016 to 2017. Euro Surveill. 2018;23(46):1800516.

15. European Centre for Disease Prevention and Control. Surveillance of antimicrobial resistance in Europe-annual report of the European Antimicrobial Resistance Surveillance Network (EARS-Net) 2017. Stockholm: ECDC; 2017. 